\title{
Die Entwicklung der Zeitschrift für Orthopädie und Unfallchirurgie - aus Sicht der Herausgeber
}

\author{
Development of the Journal "Zeitschrift für Orthopädie und Unfallchirurgie" - \\ The Editors' Point of View
}

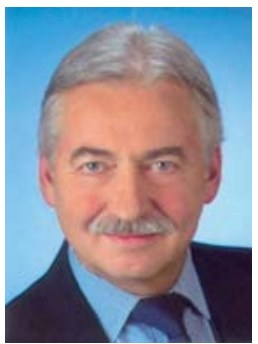

K. Weise

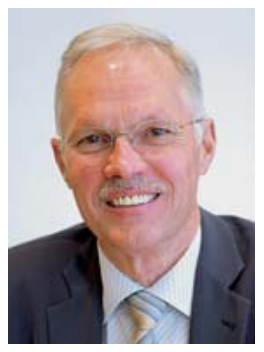

F. U. Niethard

Bibliografie

DOI $10.1055 / s-2008-1038682$ Z Orthop Unfall 2008; 146: 311

(c) Georg Thieme Verlag KG Stuttgart . New York . ISSN 1864-6697

\section{Korrespondenzadressen} Prof. Dr. med. Kuno Weise BG-Unfallklinik

Schnarrenbergstraße 95 72076 Tübingen

Tel.: 07071/606-1001

Fax: 07071/606-1002 weise@bgu-tuebingen.de

Prof. Dr. med. Fritz U. Niethard Orthopädische Klinik Universitätsklinik der RWTH Aachen

Pauwelsstraße 30

52074 Aachen

Tel.: 0241/808-94 10

Fax: 0241/808-2453

funiethard@

orthopaedie-aachen.de

\section{Liebe Leserinnen und Leser, $\nabla$}

seit gut einem Jahr gibt es die „Zeitschrift für Orthopädie und Unfallchirurgie“, eine aus retrospektiver Sicht nicht nur der verantwortlichen Herausgeber sehr gute und vorausschauende Entscheidung des Thieme Verlages. Aus der „Zeitschrift für Orthopädie und ihre Grenzgebiete“ und der „Aktuellen Traumatologie“ wurde ein Publikationsorgan, welches die Bestrebungen der Fachgesellschaften DGU und DGOOC widerspiegelt, den Buchstaben der neuen Weiterbildungsordnung gerecht zu werden und zu einer gemeinsamen Dach-/Fachgesellschaft zusammenzufinden. Auf diesem Wege ist man bereits ein beträchtliches Stück vorangekommen, wobei dieses ohne Berücksichtigung der jeweiligen Spezialitäten und Besonderheiten von Orthopädie und Unfallchirurgie nicht sinnvoll wäre. Gerade die fachspezifischen Eigenheiten machen die Attraktivität unserer Zeitschrift aus, indem Themen vom Polytrauma bis zur Säuglingshüfte angeboten und auf diese Weise informative Beiträge zum Wissensstand während der Weiterbildung für die Facharztkompetenz „Orthopädie und Unfallchirurgie“ geleistet werden. Das an die Adresse der jüngeren, in dieser Phase befindlichen Mitarbeiter gerichtete Hauptaugenmerk unserer Zeitschrift bedeutet natürlich nicht, dass „ausgewachsene Unfallchirurgen und Orthopäden“ oder Fachärzte für „Orthopädie und Unfallchirurgie" nicht in gleicher Weise Zielgruppe unseres Journals sind.

Wir, die verantwortlichen Herausgeber der „Zeitschrift für Orthopädie und Unfallchirurgie“", stellen mit Freude fest, dass das Verhalten der Kollegen bezüglich des Einreichens von Manuskripten nach der Fusion keineswegs nachgelassen, sondern eher zugenommen hat. Aufgrund unseres sehr gut funktionierenden Peer-Review-Verfahrens und der damit verbundenen Listung im Science Citation Index und anderen Institutionen existiert ein ungebrochener Anreiz, uns Originalarbeiten anzubieten, deren Inhalt unseren gehobenen Ansprüchen und dem unserer Leser in hohem Maße gerecht wird. Dass dies nunmehr fast ausschließlich auf elektronischem Wege erfolgt, entspricht der zeitgemäßen Form der Manuskriptbearbeitung. Eine bewusst große Zahl an Beiräten, die für Spezialbereiche aus Orthopädie und Unfallchirurgie eine anerkannte Expertise aufweisen, garantiert uns ein gleichermaßen faires wie fundiertes Reviewing. Die Spezialrubriken wie „CME-Refresher“ und „Operative Techniken" werden ebenso gut angenommen wie die aktuellen Nachrichten im Anfangsteil und die Kurzberichte unter dem Motto „Für Sie gelesen“. Man könnte die Bilanz über das erste Jahr gemeinsamer Herausgeberschaft und die außerordentlich gute Zusammenarbeit mit dem Verlag wie folgt ziehen:

„Es ist alles gut, machen wir einfach so weiter.“

Bekanntlich ist jedoch Stillstand Rückschritt, was bedeutet, dass wir auch zukünftig bestrebt sein wollen, unsere Zeitschrift voranzubringen und noch attraktiver zu gestalten. So wurde anlässlich der letzten Herausgebersitzung beschlossen, gerade auch die jüngere Generation von Orthopäden und Unfallchirurgen, welche die neue Facharztkompetenz anstrebt oder bereits besitzt, mehr an die ZfOU zu binden, indem wir ihnen ein Forum für die Weitergabe von Informationen, z.B. bezüglich der Erfahrungen mit der neuen WBO in den einzelnen Bundesländern oder der unbefriedigenden Situation der nachrückenden Generation und vieles andere mehr bieten.

Ganz wichtig ist uns unverändert die Werbung um interessante und aktuelle Arbeiten mit unterschiedlichen Schwerpunkten. Die verantwortlichen Herausgeber und ihre Mitarbeiter kümmern sich in der Bearbeitung jeweils um die Themen, welche ihrer eigenen Fachrichtung entsprechen. In diesem Zusammenhang wünschen wir uns einen anhaltend positiven Trend zur Einreichung orthopädisch ausgerichteter Manuskripte und eine ansteigende Zahl unfallchirurgisch gewichteter Arbeiten. Im jetzt vorliegenden Heft haben wir neben der Endoprothetik und der Infektion gerade auch dem Trauma breiteren Raum gegeben, wobei die ausgewogene Mischung unterschiedlicher Themen maßgeblich den Reiz der Zeitschrift mitbestimmt.

Die verantwortlichen Herausgeber der „Zeitschrift für Orthopädie und Unfallchirurgie " und der Thieme Verlag in Gestalt der dort für die Zeitschrift tätigen Mitarbeiter würden sich wünschen, Ihr Interesse an den angebotenen Themen möge weiterhin bestehen bleiben. Teilen Sie uns Ihre Kritik und Ihre Wünsche freimütig mit, damit uns eine stetige Verbesserung der Qualität dieses Journals ermöglicht wird. Herausgeber und Verlag danken Ihnen für Ihre nachhaltige Treue zur ZfoU. 